\title{
Are hue and saturation carried in different neural channels?
}

\author{
Sarah E. Regan, ${ }^{1, *}$ (i) Robert J. Lee, ${ }^{2}$ (i) Donald I. A. MacLeod, ${ }^{3}$ and Hannah E. Smithson ${ }^{1}$ (i) \\ ${ }^{1}$ Department of Experimental Psychology, University of Oxford, Oxford, UK \\ ${ }^{2}$ School of Psychology, University of Lincoln, Lincoln, UK \\ ${ }^{3}$ Department of Psychology, University of California, San Diego, La Jolla, California 92093, USA \\ ${ }^{*}$ Corresponding author: sarah.regan@biodtp.ox.ac.uk
}

Received 2 November 2017; revised 16 January 2018; accepted 30 January 2018; posted 30 January 2018 (Doc. ID 312522$) ;$ published 29 March 2018

\begin{abstract}
Chromatic discrimination data show that a smaller physical stimulus change is required to detect a change in hue than to detect a change in saturation [Palette 30, 21 (1968); Proc. R. Soc. London Ser. B 283, 20160164 (2016)], and, on this basis, it has been suggested that hue and saturation are carried in different neural channels [Color Space and Its Divisions: Color Order from Antiquity to the Present (Wiley, 2003), p. 311]. We used an adaptation paradigm to test explicitly for separate mechanisms, measuring hue and saturation detection thresholds before and after adaptation to hue and saturation stimuli. Within-condition adaptation did not elevate detection thresholds significantly more than between-condition adaptation. We therefore did not find psychophysical evidence for a neural channel that extracts hue thresholds more effectively than the neural channel or channels that determine saturation thresholds. () 2018 Optical Society of America
\end{abstract}

OCIS codes: (330.0330) Vision, color, and visual optics; (330.1690) Color; (330.1720) Color vision; (330.5510) Psychophysics; (330.7310) Vision; (330.7320) Vision adaptation.

https://doi.org/10.1364/JOSAA.35.00B299

\section{INTRODUCTION}

Hue and saturation are important perceptual qualities of color. Hue is the subjective quality of appearing, for example, blue, bluish-green, or green. Saturation is the subjective counterpart of colorimetric purity, where a saturated color can be desaturated to a pastel shade by adding white. It is useful both in vision science and commercially to be able to quantify colors and the perceptual relationships between them. Color spaces are models that attempt to achieve these aims.

Such spaces have been designed for a range of motivations. For example, the CIE 1931 color space was introduced to provide a common standard in science and industry for the measurement and communication of color and for the translation of physical light measurements to color percepts in a standard observer [1]. Reduction of full spectral specification of lights to a three-dimensional space rests on the trichromacy of the human visual system, which means that any visible light can be matched by adjusting the intensities of three independent primaries. Meanwhile, MacLeod-Boynton color space and Derrington-Krauskopf-Lennie (DKL) color space made representation of the underlying physiology a more central aim $[2,3]$. One axis of these spaces represents the comparison of long- and middle-wavelength ( $\mathrm{L}$ and $\mathrm{M}$ ) cone signals, thought to be implemented in the midget ganglion cells [4]. The other axis represents the inhibition of excitatory shortwavelength (S) cone signals by the summed signals of $\mathrm{L}$ and $\mathrm{M}$ cones, thought to be implemented in the small bistratified ganglion cells [5]. MacLeod-Boynton space considers only the equiluminant plane, where the sum of $\mathrm{L}$ and $\mathrm{M}$ cone signals is held constant; DKL space additionally uses a third axis to represent isochromatic variation that isolates the photopic luminance mechanism and places the white point at the origin of the space.

To represent perceptual relationships between colors, it is desirable to arrange colors in $2 \mathrm{D}$ or $3 \mathrm{D}$ Euclidean space in a perceptually isomorphic manner. For a color space to be perceptually isomorphic, any two lines of the same length must represent the same perceptual distance (i.e., the same number of just-noticeable differences). MacAdam [6] showed that, in CIE 1931 color space, the standard deviations of various isoluminant color matches constituted ellipses of varying sizes, and that there was no possible Euclidean projection that could render those ellipses circular and of equal size. Thus, despite the appeal of such a space, it is in fact impossible to construct a perceptually isomorphic Euclidean color space [7], and color 
models (e.g., CIE L*a*b*, CIE Luv) that attempt to achieve this do not do so completely.

One characteristic nonlinearity of color models is the phenomenon known as the "super-importance of hue." Nickerson measured observers' abilities to discriminate Munsell chips along dimensions equivalent to hue, saturation, and brightness and created a color formula that described the change needed to generate a step of just-noticeable difference along any dimension from any chromaticity [8]. In a Euclidean space based on this formula, the number of just-noticeable steps around the perimeter of a circle centered on the completely desaturated white point ought to be $2 \pi r$, where $r$ is the number of justnoticeable steps along the radius. In fact, Judd calculated it to be slightly more than $4 \pi r$ [9]. This means that a smaller physical stimulus change is required to detect a change in hue than to detect a change in saturation, which he called "the super-importance of hue." This particular example of the nonlinearity of color models has been taken to suggest the existence of hue channels, i.e., mechanisms encoding color differences that are tangential to circles centered on the white point [10], separate from channels representing saturation. The present experiments investigate the neural encoding of color, with specific reference to the nonlinearities implied by hue super-importance.

\section{A. Color Mechanisms}

Understanding the processing of cone responses by later stages of the visual system, and ultimately understanding the link between these processing stages and color appearance, has been a central aim of color vision research [11]. Psychophysical experiments have been used to quantify the number and nature of post-receptoral mechanisms, additionally guided by physiological and anatomical knowledge of the underlying biological substrate. The L/M-opponent and S-opponent mechanisms, represented in the MacLeod-Boynton and DKL color spaces, were described as "cardinal" mechanisms by Krauskopf $e t$ al. [12]. The special status given to these mechanisms stems from the highly selective losses of chromatic sensitivity that were produced by adaptation to a temporally varying stimulus modulated along one of the $\mathrm{S}, \mathrm{L}-\mathrm{M}$, or $\mathrm{L}+\mathrm{M}+\mathrm{S}$ axes, compared with the broader losses produced by adaptation to intermediate axes of modulation. The chromatically opponent cardinal mechanisms identified by this psychophysical method have been strongly linked with the separate populations of cells in the macaque LGN [3] that inherit their tuning from the midget and small bistratified retinal ganglion cells $[4,5]$.

Over the years, a number of higher-order color mechanisms, intermediate to the cardinal directions, have been postulated $[13,14]$. However, like the cardinal color mechanisms, they tend to be mechanisms that pass radially through the white point. For example, higher-order mechanisms have been postulated to explain the phenomenon of unique hues. The four unique hues (red, green, blue, and yellow) are those that appear phenomenologically unmixed. The after-effect of adaptation to red is green and vice versa; the after-effect of adaptation to blue is yellow and vice versa. Opponent color theory therefore argued that we have two bipolar color channels, i.e., red-green and yellow-blue [15]. It is now agreed that the poles of the chromatically opponent midget and small-bistratified ganglion cell responses do not correspond to the unique hues (although misleadingly, the L/M-opponent channel is often referred to as the red-green channel and S-opponent as the yellow-blue channel). As a result, the phenomenon of unique hues is often ascribed to a third processing stage, after the first and second stages of the photoreceptors and the chromatically opponent pathways, in which signals from the second stage are recombined to give the two unique-hue channels [16].

One potential signature of a specific higher-order mechanism is the enhanced discrimination found for thresholds along a reddish-greenish line compared with those along a bluishyellowish line $[17,18]$. This asymmetry has been linked to the color distributions of natural environments $[17,18]$, which typically extend along the daylight locus that varies between bluish and yellowish [19]. There are a number of possible underlying reasons for this association. It may be that long-term chromatic adaptation to the statistics of natural chromatic environments preferentially reduces sensitivity along the axis of maximal variation. Indeed, efficient coding suggests that, in the absence of other factors, discrimination step size should co-vary with the range of stimuli to be encoded. Additionally, if threshold minima are the signature of a sensory channel in equilibrium [20], the asymmetry may represent distinct neural channels tuned to directions intermediate to those of the cardinal mechanisms. The putative unique-hue channels, for example, may exist at a cortical locus [21], but evidence for this is weak, as physiological studies typically find narrow chromatic tuning of cortical neurons, in which all directions in color space are represented roughly equally [22]. Danilova and Mollon [20] identify reports of retinal and LGN neurons that draw synergistic inputs from $L$ and $S$ cones and an opposed input from $\mathrm{M}$ cones, which would in principle support enhanced discrimination along a reddish-greenish line.

Physiological measurements of the chromatic response of cells in retina, LGN, or early visual cortex typically show tuning that is consistent with a linear combination of cone inputs $[3,23,24]$, and, as such, these mechanisms are well described by vectors in cone space. However, later color-appearance mechanisms must depend on inherently nonlinear processes (such as retinal adaptation) and, whilst the R-G, B-Y unique-hue channels have been modeled as a linear combination of cone signals $[25,26]$, this is likely to be a substantial oversimplification.

Hue super-importance [9] and the structure of MacAdam ellipses [6] are signatures of these nonlinearities that cannot be adequately captured in a Euclidean color space [7]. Asymmetries in attentional filtering of hue and saturation also suggest a more complex metric space [27]. The MacLeodBoynton and DKL color spaces are therefore unlikely to be appropriate spaces within which to represent putative "hue" or "saturation" mechanisms or the stimuli that isolate them. Until we have a better understanding of color-appearance spaces, our ability to visualize the tuning of these mechanisms is limited.

In summary, higher-order mechanisms have most commonly been conceived as mechanisms that are tuned to a narrow range of hue angles and that respond to modulation through the white point (i.e., to changes in saturation at a fixed 
hue angle). Some of these mechanisms have possible biological correlates. However, there have been fewer discussions of hue mechanisms and no proposed biological correlate.

\section{B. Related Work}

Recently, Danilova and Mollon compared thresholds for step changes either in hue or in saturation to investigate the super-importance of hue [28]. The authors used a 4AFC discrimination task on a neutral D65 background where a briefly displayed target was differentiated from three distractors either by hue or saturation. They defined their stimuli in MacLeodBoynton color space, scaling it for individual observers by detection thresholds along the opponent axes. Reference points were selected along a +45 deg or -45 deg line in scaled MacLeod-Boynton space at six radial distances from the white point in all four quadrants of the color space. Chromaticities for the targets and distractors were also defined along a $+45 \mathrm{deg}$ or -45 deg line through the reference points; therefore, the underlying trichromatic and opponent mechanisms had the same modulation across conditions. At most distances from the white point, they replicated the super-importance of hue. Very close to the white point, there was no significant hue super-importance. However, the authors did not directly test whether hue and saturation are carried in separate neural channels.

\section{Present Study}

A classic method of determining whether a perceptual characteristic is carried in a discrete perceptual mechanism is to use an adaptation paradigm. Prolonged viewing of a stimulus can affect subsequent sensitivity to stimuli of the same or a similar kind. The degree of threshold elevation of test stimuli can indicate the extent to which they share adaptable mechanisms. Such a paradigm was responsible for the identification of the cardinal chromatic channels: The $L$ versus $M$ and $S$ versus $(\mathrm{L}+\mathrm{M})$ channels that underlie the axes of MacLeod-Boynton color space described above. Krauskopf, Williams, and Heeley presented equiluminant stimuli that were temporally modulated through the white point in color space while keeping the time-averaged stimulation of the $\mathrm{L}, \mathrm{M}$, and $\mathrm{S}$ cones constant across conditions, an innovation that allowed post-receptoral mechanisms to be targeted. Two kinds of flicker elevated detection thresholds for only the opponent combination that had been habituated. The first of these was flicker that modulated the $\mathrm{L}$ and $\mathrm{M}$ cones in opposition while holding $\mathrm{S}$ excitation constant. The second was flicker that modulated the $S$ cones while holding $\mathrm{L}$ versus $\mathrm{M}$ excitation constant. Conversely, flicker that varied in terms of a combination of both opponent mechanisms raised detection thresholds in all directions [12].

In this study, we presented stimuli that were matched in their time-averaged trichromatic and cardinal modulations, but that differed in their temporal modulation of hue and saturation. To achieve this, the stimuli modulated both of the cardinal axes over time with a triangular waveform centered on the white point; in different conditions, however, the waveform of the $S /(\mathrm{L}+\mathrm{M})$ modulation was phase-shifted relative to that of the $\mathrm{L} /(\mathrm{L}+\mathrm{M})$ modulation [see Section 2.C.2 and Fig. 1(a), left panel]. Modulation around the white point ensured that the time-averaged excitation of the trichromatic and cardinal mechanisms was matched for all stimuli; thus, any differences between conditions could truly be attributed to post-cardinal mechanisms. Different phases of combination produced modulations that were tangential or radial to the time-averaged white point, varying predominantly in hue or in saturation, respectively.

In Experiment 1, we separately collected discrimination thresholds for stimuli that modulated in hue or in saturation. In Experiment 2, we collected discrimination thresholds for hue or saturation modulations following adaptation to either (a)

(i) $0^{\circ}$ phase shift

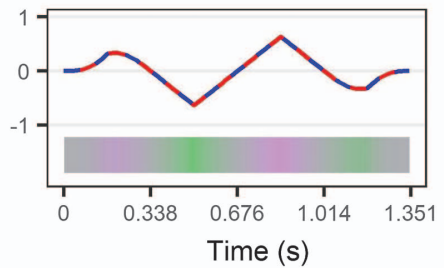

(ii) $180^{\circ}$ phase shift

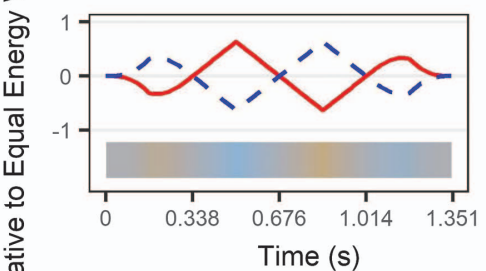

(iii) $-90^{\circ}$ phase shift

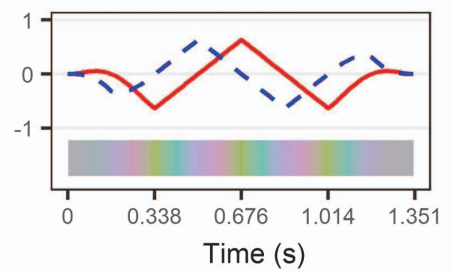

Time (s)

(iv) $90^{\circ}$ phase shift

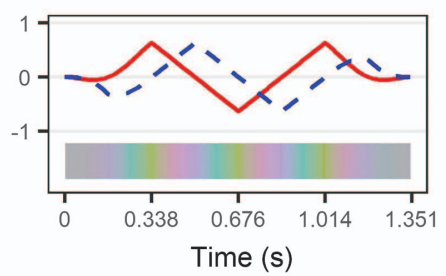

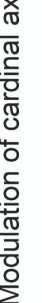

(v) diagonal right

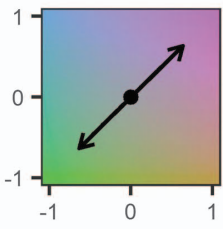

Change in $L /(L+M)$

(vi) diagonal left

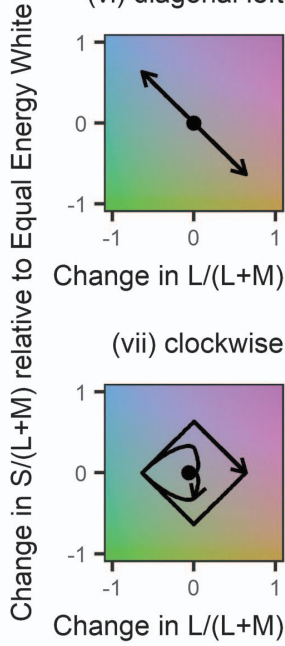

(viii) anti-clockwise

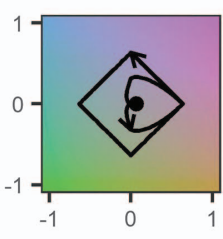

Change in $L /(L+M)$ (b)

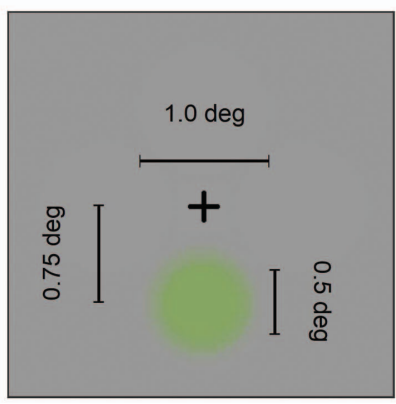

Fig. 1. (Continued) 
Fig. 1. Panel (a) shows how the stimuli are defined in terms of the cardinal mechanisms. For illustrative purposes, we use a hypothetical observer with extremely high cardinal thresholds so that the colors are clearly visible. The maximum modulation of the component mechanisms is set to $63.4 \%$ of cardinal flicker thresholds (see Discussion, Section 4.C, for why this value was chosen). Panels (a.i) to (a.iv) show the $\mathrm{L} /(\mathrm{L}+\mathrm{M})$ (solid red lines) and $\mathrm{S} /(\mathrm{L}+\mathrm{M})$ (dashed blue lines) modulations relative to equal energy white (EEW) used to create the four noncardinal flicker conditions. Each stimulus is composed of two periods of modulation; a cosine ramp has been applied to the first and last half-period. The starting phase has been randomized for each trial. Corresponding color appearance over time is inset at the bottom of each plot. Panels (a.v) to (a.viii) plot the resulting paths of the chromaticity coordinates (solid black lines with arrows) in MacLeod-Boynton space, zoomed in to the cardinal thresholds. When the phase of the $\mathrm{S} /(\mathrm{L}+\mathrm{M})$ modulation is displaced relative to $\mathrm{L} /(\mathrm{L}+\mathrm{M})$ modulation by $0 \mathrm{deg}$, the path in MacLeod-Boynton space traces out a diagonal line through the white point, slanting rightwards (a.i and a.v, respectively). When the phase shift is $180 \mathrm{deg}$, the path is a left-slanting diagonal line through white (a.ii and a.vi, respectively). When the phase shift is $-90 \mathrm{deg}$, the path is a clockwise diamond (a.iii and a.vii, respectively). When the phase shift is $90 \mathrm{deg}$, the path is a counterclockwise diamond (a.iv and a.viii, respectively). The mean chromaticity (black dots) for each stimulus is, on average, only $6.5 \%$ of maximum deviation from the white point. Panel (b) shows the spatial characteristics of the stimulus on an occasion when it has been presented in the bottom spatial quadrant, and at a moment when it appears green. Its center is displaced from the fixation point by $0.75 \mathrm{deg}$ of visual angle. The central $0.5 \mathrm{deg}$ of the stimulus is of maximum chromatic contrast for a given frame. The edge of the stimulus blurs to the background chromaticity in a cosine ramp such that the total diameter of the stimulus is $1.0 \mathrm{deg}$. See also Visualization 1, Visualization 2, Visualization 3, Visualization 4, Visualization 5, Visualization 6, and Visualization 7 for movies of the test and adaptation stimuli.

hue or saturation modulations. Thresholds following adaptation were analyzed in terms of elevation compared with nonadaptation test thresholds. If hue and saturation were carried in separate neural channels, we would expect the elevation of flicker-detection thresholds to be higher for within-condition adaptation than for between-condition adaptation. That is, threshold elevation for adapting to hue and testing hue and for adapting to saturation and testing saturation should be higher than adapting to hue and testing saturation and for adapting to saturation and testing hue.

\section{METHODS}

\section{A. Ethics}

This study was approved by the Medical Sciences Interdivisional Research Ethics Committee at the University of Oxford, in accordance with the Declaration of Helsinki.

\section{B. Apparatus}

All procedures were carried out in a dark room. Stimuli were presented on an NEC color monitor (MultiSync FP2141SB) controlled by a Cambridge Research Systems ViSaGe graphics system running in palette mode giving an 8-bit index and a 14-bit per channel RGB-encoded output value. The monitor had a spatial resolution of $800 \times 600$ pixels, and its refresh rate was set to $160 \mathrm{~Hz}$. Following data collection, we confirmed that measured thresholds were not instrumentally limited. The experiment was run from MATLAB R2011a. Staircases were controlled by the Palamedes toolbox [29]. The monitor was gamma-corrected with a Cambridge Research Systems ColorCAL II colorimeter, and the spectral energy outputs of the CRT phosphors were measured by a Cambridge Research Systems SpectroCAL MKII spectral radiometer. Background luminance was confirmed using the ColorCAL. Viewing distance was maintained by a chin and forehead rest positioned $92 \mathrm{~cm}$ from the CRT monitor. Observers viewed the stimuli binocularly.

\section{Stimuli}

\section{Color Space}

All chromaticity coordinates were defined in terms of their location on a MacLeod-Boynton diagram [2], as constructed by multiplying the Stockman and Sharpe $2 \mathrm{deg}$ cone fundamentals (scaled so that $L(\lambda)+M(\lambda)$ is equal to the luminosity function $V^{*}(\lambda)$ and that $S(\lambda)$ peaks at unity [30]) by the spectral energy output of the CRT phosphors. All chromaticity coordinates were defined relative to the coordinates of equal energy white $(\mathrm{EEW})$ at $14 \mathrm{~cd} / \mathrm{m}^{2}$.

\section{Chromatic Modulation}

All temporal chromatic modulation was based on triangle-wave deviation from the coordinates of EEW along a given axis of the MacLeod-Boynton diagram [see Fig. 1(a)]. Triangle waves were selected rather than sine waves so that the wave spends the same amount of time at all cardinal contrasts, which would not be possible with waves that do not have gradients of a constant magnitude, such as sine waves.

Where the test stimulus was presented briefly for chromatic threshold detection (see Sections 2.E.2, 2.E.3, and 2.E.4), two cycles were presented, and the starting phase was randomized. Where variation in terms of only one of the cardinal color mechanisms $\mathrm{L} /(\mathrm{L}+\mathrm{M})$ and $\mathrm{S} /(\mathrm{L}+\mathrm{M})$ was desired (see Section 2.E.2), coordinates of one axis were kept constant at the value of EEW, while those of the other axis were temporally modulated in a triangle wave.

Stimuli that varied predominantly in terms of saturation were created by shifting the phase of $\mathrm{S} /(\mathrm{L}+\mathrm{M})$ modulation relative to $\mathrm{L} /(\mathrm{L}+\mathrm{M})$ modulation by either $0 \mathrm{deg}$ or $180 \mathrm{deg}$ (such that the locus of chromaticities visited lay on a diagonal line passing through EEW on the MacLeod-Boynton diagram, slanted right or left, respectively). Stimuli that varied predominantly in terms of hue were created by shifting the phase of $\mathrm{S} /(\mathrm{L}+\mathrm{M})$ modulation relative to $\mathrm{L} /(\mathrm{L}+\mathrm{M})$ modulation by either $-90 \mathrm{deg}$ or $+90 \mathrm{deg}$ (tracing out a diamond centered on EEW on the MacLeod-Boynton diagram in the clockwise or counterclockwise direction, respectively).

If the stimuli were allowed to modulate forever, the average chromaticity in all cases would be EEW. However, our test stimuli were presented briefly. In the case of hue, there was no phase at which the chromaticity was EEW, and sudden saturation changes from and to EEW at the start and end of the stimulus presentation might facilitate detection. Therefore, for all stimulus types, the starting phase was randomized, and a 
cosine ramp was applied to the first half-period and the last half-period of each two-period stimulus such that the stimulus began and ended at the coordinates of EEW and ramped smoothly up to and down from the maximum contrast of the component modulations. The ramped onsets and offsets of the modulations did cause the mean chromaticity on each trial to be slightly offset from the white point. However, the deviation of the mean chromaticity from EEW on each trial was not large (never more than $10.1 \%$, and on average $6.5 \%$, of the maximum contrast on any given trial), which ought to be imperceptible given that the maximum contrast was generally at or around threshold.

\section{Temporal Properties}

The hue and saturation stimuli modulated at $1.48 \mathrm{~Hz}$ (each cycle was specified over 108 frames at the frame rate of $160 \mathrm{~Hz}$ ). For the adaptation stimuli, modulations continued for $2 \mathrm{~min}$ at the start of each session, with $6 \mathrm{~s}$ of top-up adaptation on each trial. For test stimuli, two cycles were presented in $1.35 \mathrm{~s}$, with a $0.338 \mathrm{~s}$ ramped onset and offset, and peak contrast presented for 0.675 s. Hue stimuli cycled through the full range of hue-angles. They also necessarily differed from the background in saturation, and, for test stimuli, the ramp additionally introduced a slow saturation modulation. Saturation stimuli varied in their proximity to the white point, with two saturation minima per cycle. They included a change in hue at the two poles of the excursion. Therefore, the "hue" stimuli were not exclusively modulations of hue, and the "saturation" stimuli were not exclusively modulations of saturation. However, the stimulus design offered substantially different modulations to the putative hue and saturation mechanisms while ensuring that hue and saturation stimuli were perfectly matched in the modulation they offered to the cardinal mechanisms.

\section{Spatial Arrangement}

The spatial layout of the stimulus is presented in Fig. 1(b). All stimuli were discs with a blurred edge to limit use of edge cues. Each stimulus had a central region of maximum saturation $0.5 \mathrm{deg}$ of visual angle in diameter, with an additional $0.5 \mathrm{deg}$ in diameter of blur into the background chromaticity, where the proportion of saturation from center to background was defined by a cosine function. Stimuli were centered $0.75 \mathrm{deg}$ above, below, to the left, or right of a fixation cross such that the central $0.5 \mathrm{deg}$ of all stimuli fell within the smallest typical area of macular pigment, which is 2 deg across in 18-year-olds [31] and avoided the blind spot, 15 deg nasally medial to the fovea, and the S-cone free area in the central $0.4 \mathrm{deg}$ of the fovea [32]. These locations will hereafter be referred to as the top, bottom, left, and right spatial quadrants.

\section{Task}

For E.2 Pre-Experiment 2, E.3 Experiment 1, and E.4 Experiment 2, triangle-wave flicker-detection thresholds were measured for the relevant conditions with the luminance adjustments obtained from heterochromatic flicker photometry (HFP; see Section 2.E.1). Each trial was four-alternative spatial forced-choice, presenting a stimulus modulating at $1.48 \mathrm{~Hz}$ in one of the four spatial quadrants on a $14 \mathrm{~cd} / \mathrm{m}^{2}$ background metameric to EEW. Observers adapted for 2 min to the background. Observers were then asked to look steadily at the fixation cross and report the stimulus location by pressing buttons on a response box.

Modulation amplitude on each trial was determined by two interleaved 25-trial Palamedes QUEST staircases with a prior mean and SD obtained in pilot testing. The trial and final threshold chromatic contrasts were the mean of the posterior probability density function [33]. Because perceptual space is logarithmic [34], modulation amplitude varied on a log scale.

The order of conditions was counterbalanced. Thresholds became lower with practice; therefore, to ensure observers had adequately learned to do the task before measurements were taken, all observers completed at least one practice run, following which the final threshold for each of the two conditions was the mean of the last six runs after the observer had reached an asymptotic level of performance for each.

\section{E. Procedures}

\section{Pre-Experiment 1: Heterochromatic Flicker Photometry}

Equiluminant stimuli in different parts of the MacLeodBoynton diagram were derived via subjective HFP [35] to control for individual differences in spectral sensitivity of the luminance mechanism. HFP was carried out on the CRT monitor described above. Chromatic modulation constituted a triangle wave along one of the two cardinal axes, and relative luminance of the poles was adjusted until flicker was minimal. In order for the observers to not match the appearance of the flickering stimulus to the background, the background was black. Prior to the staircase, observers adapted for $2 \mathrm{~min}$ to a $14 \mathrm{~cd} / \mathrm{m}^{2}$ background metameric to EEW. To limit rod intrusion to the levels that would occur in the threshold measurements, staircases were brief, and each setting was preceded by a $10 \mathrm{~s}$ re-adaptation period to the background. Although little adjustment should be necessary for $S /(L+M)$ variation, HFP was performed for chromatic temporal modulation along both the $\mathrm{L} /(\mathrm{L}+\mathrm{M})$ and the $\mathrm{S} /(\mathrm{L}+\mathrm{M})$ axis. Individual measurements were carried out for each of the four spatial quadrants in the spatial arrangement described above due to the possibility of retinal heterogeneity [36].

Observers were asked to look at a fixation cross and respond to the apparent flicker of one spatial quadrant. Constantchromaticity stimuli metameric to EEW were placed in the three spatial quadrants that did not contain a flickering stimulus to facilitate steady fixation. The flicker frequency was $16 \mathrm{~Hz}$, which is lower than typical HFP but suitable for the lower luminances achieved by a CRT monitor [37]. Maximum chromatic deviation was set to three times the estimated threshold from pilot tests. Observers pressed up and down buttons on a response box to increase the luminance of one pole of the chromatic axis while decreasing the other on an up-down staircase with seven reversals, where the point of least flicker was defined as the mean of the final four reversals. They were instructed to explore the range of flicker until they had "changed direction" in the flicker space twice, at which point they should move back and forth in the zone of no apparent flicker, changing direction when and only when the flicker began to increase. Following two practice runs, observers completed this exercise 
six separate times for each of the four spatial quadrants for each axis, and the mean of those six runs for each quadrant was taken to be the luminance correction.

It was important to ensure that the corrections resulting from the HFP settings made along the cardinal axes could successfully generalize to equate the apparent luminances of chromaticities that did not lie along the cardinal axes. We therefore applied the corrections to intermediate chromaticity pairs and showed them to the observers, flickering in counterphase at $16 \mathrm{~Hz}$, before proceeding with the next stage of the experiment. The resulting stimuli appeared to be of steady luminance, indicating that the corrections did generalize to non-cardinal chromaticities.

\section{Pre-Experiment 2: Cardinal Axis Scaling}

With the luminance adjustments obtained from Section 2.E.1 HFP and the task described in Section 2.D Task, triangle-wave flicker-detection thresholds were measured for the chromatic cardinal axes. The staircases were run in log steps in MacLeodBoynton space.

\section{Experiment 1: Phase-Shifted Non-Cardinal Thresholds}

The cardinal flicker thresholds obtained in Section 2.E.2 were used to scale chromatic modulation along both axes such that the maximum deviation from EEW of the triangle wave of each chromatic axis was the same proportion of its cardinal flicker threshold. The staircases were therefore run in $\log$ fractions of cardinal threshold. Flicker detection thresholds were measured for chromatic modulations of saturation (phase shifts of $0 \mathrm{deg}$, diagonal right; $180 \mathrm{deg}$, diagonal left) and of hue (phase shifts of -90 deg, clockwise; 90 deg, counterclockwise; see Fig. 1(a)) using the task described in Section 2.D Task. Contrast of component modulations varied in terms of log threshold units.

\section{Experiment 2: Adaptation}

Using the cardinal scaling as obtained in Section 2.E. 1 and the task described in Section 2.D, we gathered hue and saturation thresholds following adaptation to hue and saturation stimuli. To reduce the number of conditions, we used only 0 deg phase shift (diagonal right) for saturation modulation and $-90 \mathrm{deg}$ phase shift (clockwise) for hue modulation. Therefore, the four conditions were as follows: adaptation to diagonal-right saturation modulation, test diagonal-right saturation modulation; adaptation to diagonal-right saturation modulation, test clockwise hue modulation; adaptation to clockwise hue modulation, test diagonal-right saturation modulation; and adaptation to clockwise hue modulation, test clockwise hue modulation. As before, the background was metameric to EEW at $14 \mathrm{~cd} / \mathrm{m}^{2}$. Instead of beginning each session with 2 min of adaptation to the background, the observer adapted to a disc modulating at $1.48 \mathrm{~Hz}$ in terms of either hue or saturation, whose central region of maximum saturation was $12 \mathrm{deg}$ in visual angle in diameter with an additional 3 deg in diameter of blur into the background chromaticity where the proportion of contrast from center to background was defined by a cosine function. The chromatic contrast of the adaptation modulation was the maximum that could be achieved within the gamut of the monitor, which was approximately $2.45 \mathrm{log}$ threshold units above the pilot thresholds for hue and saturation. Each trial was preceded by $6 \mathrm{~s}$ of top-up adaptation with the same stimulus.

\section{F. Observers}

Four female and two male observers with normal or correctedto-normal acuity and no known color vision deficiencies completed the experiment. Observers A and B are authors.

\section{RESULTS}

\section{A. Experiment 1: Phase-Shifted Non-Cardinal Thresholds}

The mean nonadaptation test detection thresholds for individual observers and across observers are shown in Fig. 2. The mean nonadaptation saturation thresholds and mean nonadaptation hue thresholds are shown in Fig. 3. Thresholds are given as the log fraction of the individual observer's cardinal threshold (that is, log threshold units). Any negative value in log threshold units indicates that the threshold is lower than the individual observer's cardinal threshold. In two runs of one condition in one observer, the staircase did not converge to a stable position in the number of trials available, and the data points were excluded.

Mauchly's test of sphericity was violated $(p=.022)$, and the standardized residuals were not all normally distributed (Shapiro-Wilk test of normality, $p=.031$ for diagonal left), so a repeated-measures ANOVA could not be run. Instead, a nonparametric Friedman test was run with four groups: diagonal right, diagonal left, clockwise, and counterclockwise.

There was a statistically significant difference in log threshold depending on the phase of combination of the $\mathrm{L} /(\mathrm{L}+\mathrm{M})$ and $S /(\mathrm{L}+\mathrm{M})$ modulation, $\chi^{2}(3)=11.400, p=.010$. Therefore, post-hoc tests could be carried out. The assumption of symmetrical distributions was also violated for diagonal right and diagonal left (Shapiro-Wilk $p=.013$ ) and diagonal left and clockwise $(p=.027)$, so sign rank tests were used; Bonferroni corrections set the two-tailed significance level at $p<0.008$. None of the differences survived the Bonferroni corrections (diagonal right and diagonal left, $p=.031$; diagonal right and clockwise, $p=.031$; diagonal right and counterclockwise, $p=.031$; diagonal left and clockwise, $p=1.000$; diagonal left and counterclockwise, $p=.687$; clockwise and counterclockwise, $p=.687$ ).

Our primary hypothesis concerned the difference between hue thresholds and saturation thresholds. The Shapiro-Wilk test indicated that the combined hue and saturation thresholds were normally distributed (saturation, $p=.912$; hue, $p=.891$ ), so a paired t-test was run. The mean hue thresholds $(-0.424 \log$ threshold units) were higher than mean saturation thresholds (-.493) contrary to hue-super importance; however, the difference between the two thresholds did not prove significant $(p=.163)$.

\section{B. Experiment 2: Adaptation}

The mean post-adaptation test detection thresholds for individual observers and across observers are shown in Fig. 4. The mean within-condition and between-condition adaptation thresholds are shown in Fig. 5. Thresholds are given in terms of their elevation compared with nonadaptation test thresholds, again as a $\log$ fraction of the individual observer's cardinal 
(a) Mean

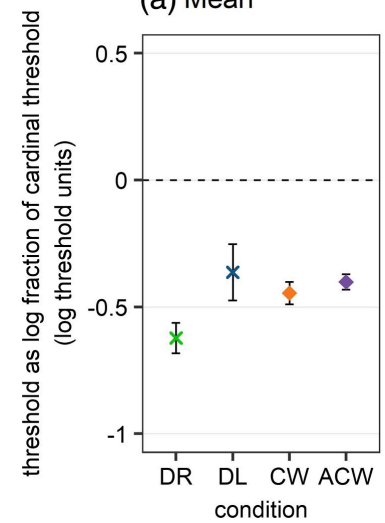

(b) Individual thresholds of 6 participants

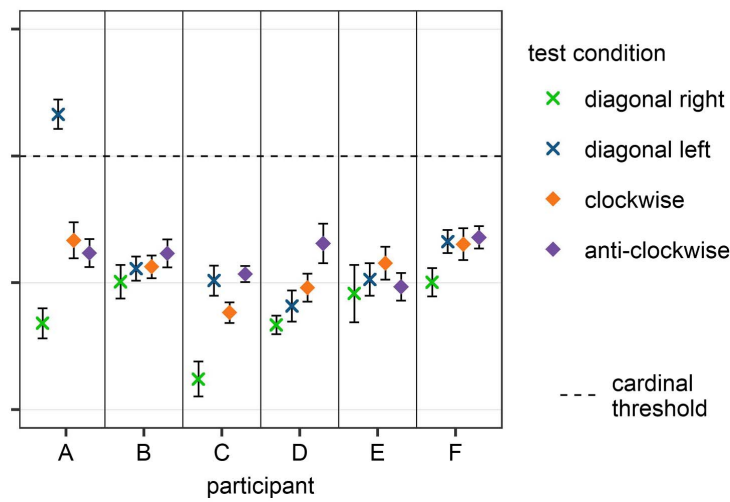

Fig. 2. Detection thresholds in log threshold units (log fraction of the individual observer's cardinal threshold, black dashed line), for phase shifts of 0 deg diagonal right (in print, light gray crosses; online, green crosses), 180 deg diagonal left (in print, dark gray crosses; online, blue crosses), -90 deg clockwise (in print, light gray diamonds; online, orange diamonds) and 90 deg counterclockwise (in print, dark gray diamonds; online, purple diamonds). Panel (a) plots the mean results across observers for Experiment 1; the error bars (black lines) represent the standard error across observers. Panel (b) plots the thresholds for each individual observer; the error bars (black lines) represent the standard error across runs. There was a statistically significant effect of phase of combination of $\mathrm{S} /(\mathrm{L}+\mathrm{M})$ modulation with $\mathrm{L} /(\mathrm{L}+\mathrm{M}$ modulation $(p=.010)$ for the four phase combinations; however, none of the pairwise comparisons were significant after Bonferroni corrections. The significant effect of phase could have been driven by the lower thresholds for 0 deg diagonal right compared with the other conditions for every observer.

(a) Mean



(b) Individual thresholds of 6 participants

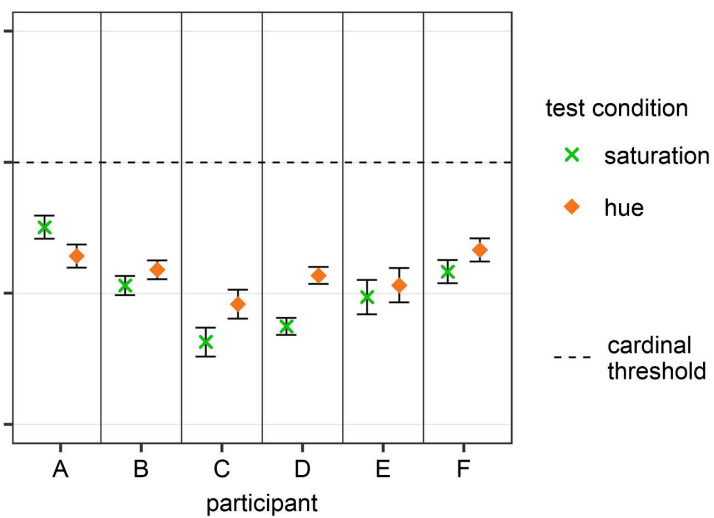

Fig. 3. Detection thresholds in log threshold units (log fraction of the individual observer's cardinal threshold, black dashed line), expressed in terms of the mean thresholds for saturation and hue, where mean saturation threshold (in print, light gray crosses; online, green crosses) is the mean of 0 deg diagonal right and $180 \mathrm{deg}$ diagonal left, and mean saturation threshold (in print, light gray diamonds; online, orange diamonds) is the mean of -90 deg clockwise and 90 deg counterclockwise. Panel (a) plots the mean thresholds across observers; the error bars (black lines) represent the standard error across observers. Panel (b) plots the individual thresholds; the error bars (black lines) represent the standard error across runs. A paired difference test showed no significant difference between hue and saturation thresholds $(p=.163)$.

threshold. In one run of one condition in one observer, the staircase did not converge to a stable position in the number of trials available, and the data point was excluded.

If hue and saturation were carried in separate neural channels, we would expect within-condition adaptation to raise thresholds more than between-condition adaptation. The Shapiro-Wilk test of normality showed that the withincondition thresholds were not normally distributed $(p=.005)$, but the distributions were symmetrical (Shapiro-Wilk for within-between, $p=.349$ ). Therefore, a Wilcoxon signed-rank two-tailed test was run. Although median within-condition adaptation thresholds (0.252 log threshold elevation) were higher than median between-condition thresholds $(0.176 \log$ threshold elevation), the difference was not significant $(p=.075)$.

\section{DISCUSSION}

Thresholds for hue differences have previously been found to be lower than those for saturation differences [9,28]. Some have cited this as evidence for separate neural channels for hue and saturation [10]. In this study, we directly tested the hypothesis that hue and saturation are carried in different neural channels. 
(a) Mean

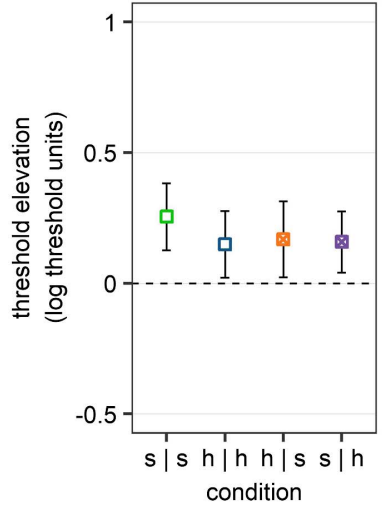

(b) Individual thresholds of 6 participants

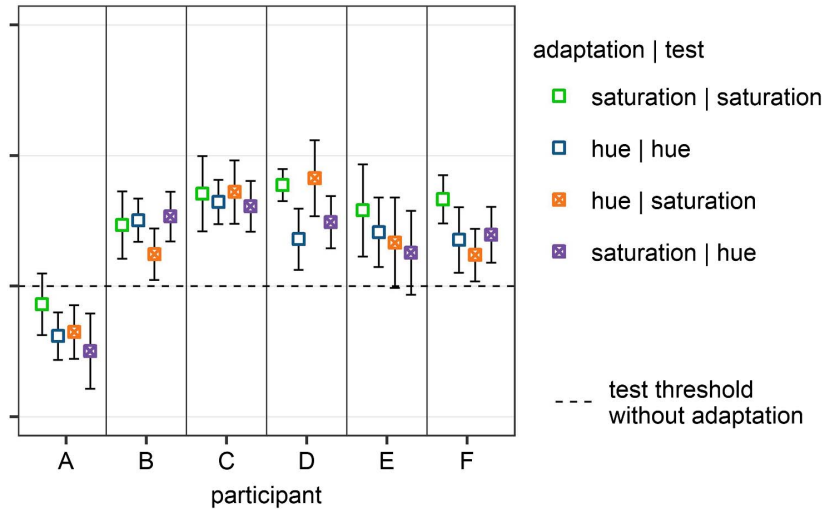

Fig. 4. Post-adaptation detection thresholds, in log threshold elevation (threshold after adaption minus threshold without adaptation, black dashed line, in log threshold units), for the following conditions: adaptation to diagonal-right saturation modulation, test diagonal-right saturation modulation (in print, light gray open squares; online, green open squares); adaptation to clockwise hue modulation, test clockwise hue modulation (in print, dark gray open squares; online, blue open squares); adaptation to clockwise hue modulation, test diagonal-right saturation modulation (in print, light gray crossed-through squares; online, orange crossed-through squares); and adaptation to diagonal-right saturation modulation, test clockwise hue modulation (in print, dark gray crossed-through squares; online, purple crossed-through squares). Panel (a) plots the mean results across observers for Experiment 2; because the data are presented in terms of the threshold elevation from pre-adaptation threshold, the error bars (black lines) represent the sum of the standard error across observers for the post-adaptation threshold and the standard error across observers for the corresponding pre-adaptation threshold. Panel (b) plots the post-adaptation detection thresholds for each individual observer; the error bars (black lines) represent the sum of the standard error across runs for the post-adaptation threshold and the standard error across runs for the corresponding pre-adaptation threshold.

(a) Mean

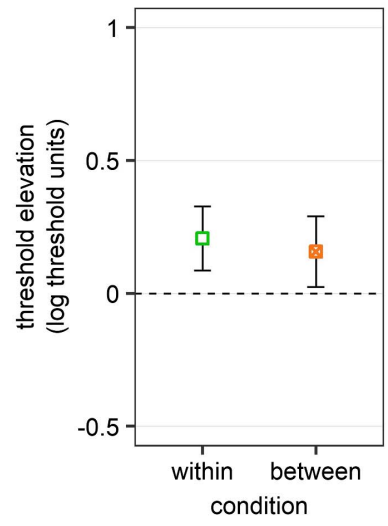

(b) Individual thresholds of 6 participants

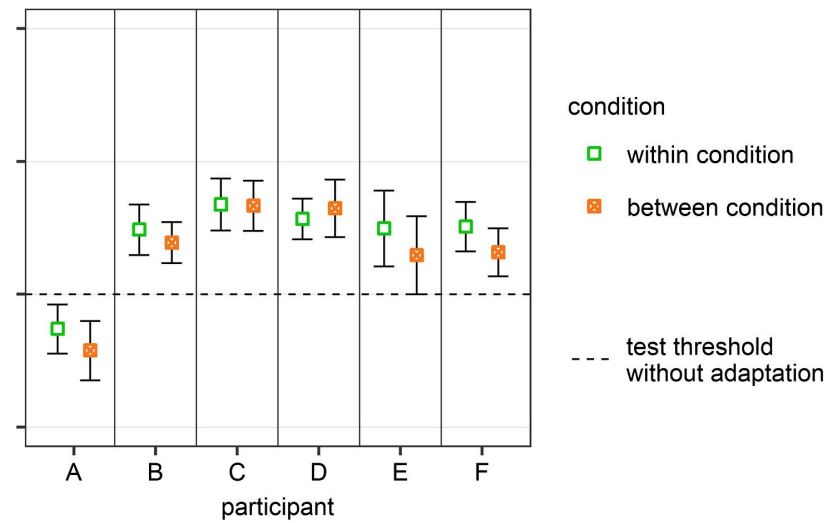

Fig. 5. Post-adaptation detection thresholds, in log threshold elevation (threshold after adaption minus threshold without adaptation, black dashed line, in log threshold units), expressed in terms of the means of within-condition thresholds and between-condition thresholds, where mean within-condition threshold (in print, light gray open squares; online, green open squares) is the mean of adaptation to diagonal-right saturation modulation, test diagonal-right saturation modulation and adaptation to clockwise hue modulation and test clockwise hue modulation; and mean between-condition threshold (in print, light gray crossed-through squares; online, orange crossed-through squares) is the mean of adaptation to hue modulation, test saturation modulation and adaptation to saturation modulation, test hue modulation. Panel (a) plots the mean threshold elevations across observers; the error bars (black lines) represent the sum of the standard error across observers for the post-adaptation threshold and the standard error across observers for the mean of the corresponding pre-adaptation thresholds. Panel (b) plots the individual threshold elevations; the error bars (black lines) represent the sum of the standard error across runs for the post-adaptation threshold and the standard error across runs for the mean of the corresponding pre-adaptation thresholds. A paired difference test showed no significant difference between hue and saturation thresholds $(p=.069)$.

\section{A. Super-Importance of Hue}

Color spaces are subject to a number of striking perceptual nonlinearities. One of these is the super-importance of hue: When just-noticeable difference steps are measured along the
Munsell hue and saturation scales, Judd indicated that there are at least twice as many just-noticeable steps in traversing the hue circle between any two color points on either side of the desaturated center as would be expected from Euclidean geometry [9]. 
A plausible basis for hue super-importance is that a circuit of the hue circle recruits a succession of many hue-selective signals that wax and wane in turn and thus follows a relatively long and complex trajectory in a high-dimensional space, where each such signal is represented by its own axis. A saturation modulation, on the other hand, modulates only a single hue signal (or only two if the complement is considered as separate).

The results of our Fig. 3, however, provide no evidence for the super-importance of hue under threshold conditions: instead, relative sensitivity to changes of saturation and hue is broadly consistent with a Euclidean model in which the isoluminant colors occupy a flat surface. There is no strict contradiction here because, if color differences conform to the simple Euclidean prediction for the small modulations associated with our threshold measurements, they could still show deviations when larger color differences are considered; in Section 4.2 below, however, we qualify this suggestion.

If the only threshold-determining chromatic channels were those modeled by the cardinal axes, the relative phases of the axes' modulations should not affect the resulting thresholds. In our experiment, we found that saturation thresholds were lower than hue thresholds, although not significantly. This replicates the findings of Danilova and Mollon, who did not find a significant advantage for hue over saturation close to the white point [28].

When thresholds for hue and saturation were analyzed separately as diagonal right, diagonal left, clockwise, and counterclockwise (see Fig. 2) with a Friedman test, we did find a significant effect of relative phase of combination; however, none of the post-hoc tests survived Bonferroni corrections. Inspection of the data indicates that the significance of the effect of relative phase was driven by the lower thresholds obtained for diagonal right compared with all other conditions. These results echo those of Fig. 6 in Danilova and Mollon: for quadrants 1 and 3 of the MacLeod-Boynton diagram, which correspond to our diagonal right, saturation thresholds may actually be lower than hue thresholds when very near the white point. They also found the difference to be nonsignificant [28].

Lack of difference between thresholds does not disprove the hypothesis that hue and saturation are carried in different neural channels. We therefore carried out an adaptation experiment to directly test this possibility.

\section{B. Separate Neural Channels for Hue and Saturation?}

We directly tested the hypothesis that hue and saturation are carried in different neural channels with an adaptation paradigm. If hue and saturation were carried in separate neural channels, we would expect within-condition adaptation to raise thresholds more than between-condition adaptation. Furthermore, as Judd showed that Nickerson's measurements predicted an extremely large advantage for hue thresholds over saturation thresholds $[8,9]$, the suprathreshold hue adaptation stimulus used in Experiment 2 should be a strong adapting stimulus. However, we did not find a significant difference between within- and between-condition adaptation. We have therefore found no evidence for separate neural channels for hue and saturation. Moreover, the results provide no support for hue super-importance at suprathreshold levels, when the suprathreshold stimuli are considered in terms of their adapting effects rather than the judged magnitudes of clearly visible color differences.

\section{Detection by Cardinal Mechanisms}

If changes in hue angle of the stimulus were detected by a (highly sensitive, nonlinear) hue channel, we would expect hue and saturation thresholds to be different. However, hue and saturation thresholds did not significantly differ from one another, which is consistent with performance based on excitation of only the cardinal mechanisms or linear combinations of these mechanisms.

One explanation of our results that we would want to rule out is that detection of the hue and saturation stimuli was in fact supported by only one of the cardinal mechanisms. If this were the case, we would expect the relative phase of the two modulations not to affect thresholds. This is indeed what we see: With the exception of the Friedman test for the four detection thresholds, all differences between thresholds were nonsignificant. However, the measured hue and saturation thresholds are lower than the cardinal thresholds measured in isolation: The mean linear fraction across conditions and observers is 0.634 linear threshold units [as shown in Fig. 1(a)]. This indicates that more than one mechanism is involved in the detection of hue and saturation stimuli.

The simplest account of the detection of our hue and saturation stimuli is that the two cardinal mechanisms detect them independently of one another. Because there are two of them, it is more likely that one or the other will meet the criterion for detection on any given trial, so the resulting thresholds are lower than cardinal thresholds (values less than zero in Figs. 2 and 3). In the case of our saturation stimuli, the two cardinal mechanisms would simultaneously but independently have the opportunity to detect the stimulus. In the case of our hue stimuli, the stimuli may stimulate first one cardinal mechanism than the other. While it is not impossible that stimulation of one cardinal mechanism facilitates detection by the other, this seems unlikely given that the final thresholds are substantially below the thresholds for each of the mechanisms.

Therefore, cardinal threshold scaling is sufficient to allow both cardinal mechanisms to contribute to detection, but, even so, we find little effect of the phase of their combination. There is no evidence that the dynamic change in hue is being detected or for the excitation of any mechanism other than the cardinal mechanisms or linear combinations of these mechanisms.

\section{Correlated Neural Noise}

If hue and saturation are not carried in separate neural channels, an alternative explanation is required for the differences in hue and saturation thresholds that have previously been found away from the white point $[9,28]$. Danilova and Mollon have advanced an alternative hypothesis: that of correlated neural noise [28]. The firing of small bistratified cells, which are responsible for the $\mathrm{S} /(\mathrm{L}+\mathrm{M})$ cardinal mechanism [5], is correlated with that of spatially close midget ganglion cells, which are responsible for the $\mathrm{L} /(\mathrm{L}+\mathrm{M})$ cardinal mechanism $[4,38]$. Correlated neural noise would make it easier to discriminate between hue stimuli, which would lie obliquely with the axis 
of correlation, than between saturation stimuli, which would lie along that axis [28].

\section{E. Conclusions}

Previous work has found striking differences between hue and saturation thresholds [9,28]. Although these threshold differences had sometimes been attributed to separate neural channels [10], no one had directly tested this hypothesis. An adaptation paradigm, the classic test of whether perceptual mechanisms can be attributed to discrete neural populations [12], yielded no evidence of separate channels for hue and saturation.

Funding. Biotechnology and Biological Sciences Research Council (BBSRC) (BB/M011224/1).

Acknowledgment. We thank J. S. Harvey and R. C. White, who provided comments on a draft of this paper.

\section{REFERENCES}

1. T. Smith and J. Guild, "The C.I.E. colorimetric standards and their use," Trans. Opt. Soc. 33, 73-134 (1931).

2. D. I. A. MacLeod and R. M. Boynton, "Chromaticity diagram showing cone excitation by stimuli of equal luminance," J. Opt. Soc. Am. 69, 1183-1186 (1979).

3. A. M. Derrington, J. Krauskopf, and P. Lennie, "Chromatic mechanisms in lateral geniculate nucleus of macaque," J. Physiol. 357, 241-265 (1984).

4. B. B. Lee, J. Kremers, and T. Yeh, "Receptive fields of primate retinal ganglion cells studied with a novel technique," Vis. Neurosci. 15, 161-175 (1998).

5. D. M. Dacey and B. B. Lee, "The 'blue-on' opponent pathway in primate retina originates from a distinct bistratified ganglion cell type," Nature 367, 731-735 (1994).

6. D. L. MacAdam, "Visual sensitivities to color differences in daylight," J. Opt. Soc. Am. 32, 247-273 (1942).

7. I. Farup, "Hyperbolic geometry for colour metrics," Opt. Express 22, 12369-12378 (2014).

8. D. Nickerson, "The specification of color tolerances," Textile Res. 6 , 505-514 (1936)

9. D. B. Judd, "Ideal color space. II. The super-importance of hue differences and its bearing on the geometry of color space," Palette 30, 21-28 (1968).

10. R. G. Kuehni, "From color-matching error to large color differences," in Color Space and Its Divisions: Color Order from Antiquity to the Present (Wiley, 2003), Chap. 8, pp. 311-335.

11. A. Stockman and D. H. Brainard, "Color vision mechanisms," in The Optical Society of America Handbook of Optics, M. Bass, C. DeCusatis, J. Enoch, V. Lakshminarayanan, G. Li, C. Macdonald, V. Mahajan, and E. V. Stryland, eds., 3rd ed. (McGraw-Hill, 2010), Chap. 11, pp. 11.1-11.104.

12. J. Krauskopf, D. R. Williams, and D. W. Heeley, "Cardinal directions of color space," Vision Res. 22, 1123-1131 (1982).

13. J. Krauskopf, D. R. Williams, M. B. Mandler, and A. M. Brown, "Higher order color mechanisms," Vision Res. 26, 23-32 (1986).

14. R. T. Eskew, "Higher order color mechanisms: a critical review," Vision Res. 49, 2686-2704 (2009).
15. E. Hering, Zur Lehre vom Lichtsinne (Gerold, 1878).

16. J. D. Mollon and G. Jordan, "On the nature of unique hues," in John Dalton's Colour Vision Legacy (CRC Press, 1997), p. 381-392.

17. K. C. McDermott and M. A. Webster, "Uniform color spaces and natural image statistics," J. Opt. Soc. Am. A 29, A182-A187 (2012).

18. J. M. Bosten, R. D. Beer, and D. I. A. MacLeod, "What is white?" J. Vis. 15(16), 5 (2015)

19. S. M. C. Nascimento, K. Amano, and D. H. Foster, "Spatial distributions of local illumination color in natural scenes," Vision Res. 120, 39-44 (2016).

20. M. Danilova and J. Mollon, "Foveal color perception: minimal thresholds at a boundary between perceptual categories," Vision Res. 62 162-172 (2012).

21. I. Abramov and J. Gordon, "Color appearance: on seeing red-or yellow, or green, or blue," Annu. Rev. Psychol. 45, 451-485 (1994).

22. P. Lennie, J. Krauskopf, and G. Sclar, "Chromatic mechanisms in striate cortex of macaque," J. Neurosci. 10, 649-669 (1990).

23. H. Sun, H. E. Smithson, Q. Zaidi, and B. B. Lee, "Specificity of cone inputs to macaque retinal ganglion cells," J. Neurophysiol. 95 837-849 (2006).

24. S. G. Solomon and P. Lennie, "The machinery of colour vision," Nat. Rev. Neurosci. 8, 276-286 (2007).

25. D. H. Krantz, "Color measurement and color theory: I. Representation theorem for Grassmann structures," J. Math. Psych. 12, 283-303 (1975).

26. D. H. Krantz, "Color measurement and color theory: II Opponentcolors theory," J. Math. Psych. 12, 304-327 (1975).

27. P. Sun, C. Chubb, C. E. Wright, G. Sperling, J. Hochberg, and R. M. Shiffrin, "Human attention filters for single colors," Proc. Natl. Acad. Sci. USA 113, E6712-E6720 (2016).

28. M. V. Danilova and J. D. Mollon, "Superior discrimination for hue than for saturation and an explanation in terms of correlated neural noise," Proc. R. Soc. London Ser. B 283, 20160164 (2016).

29. N. Prins and F. A. A. Kingdom, "Palamedes: MATLAB routines for analyzing psychophysical data" (2009), http://www.palamedestoolbox.org.

30. A. Stockman and L. T. Sharpe, "The spectral sensitivities of the middle- and long-wavelength-sensitive cones derived from measurements in observers of known genotype," Vision Res. 40, 1711-1737 (2000).

31. S. F. Chen, Y. Chang, and J. C. Wu, "The spatial distribution of macular pigment in humans," Curr. Eye Res. 23, 422-434 (2001).

32. C. A. Curcio, K. A. Allen, K. R. Sloan, C. L. Lerea, J. B. Hurley, I. B. Klock, and A. H. Milam, "Distribution and morphology of human cone photoreceptors stained with anti-blue opsin," J. Comp. Neurol. 312, 610-624 (1991).

33. P. E. King-Smith, S. S. Grigsby, A. J. Vingrys, S. C. Benes, and A. Supowit, "Efficient and unbiased modifications of the QUEST threshold method: Theory, simulations, experimental evaluation and practical implementation," Vision Res. 34, 885-912 (1994).

34. G. T. Fechner, Elements of Psychophysics [Original work published 1860] (Holt, Rinehart \& Winston, 1966), Vol. 1.

35. J. Pokorny, V. C. Smith, and M. Lutze, "Heterochromatic modulation photometry," J. Opt. Soc. Am. A 6, 1618-1623 (1989).

36. G. Wyszecki and W. S. Stiles, "The eye," in Color Science: Concepts and Methods, Quantitative Data and Formulas, G. Wyszecki and W. S. Stiles, eds., 2nd ed. (Wiley, 1982), Chap. 2, pp. 83-116.

37. J. D. Moreland, A. G. Robson, and J. J. Kulikowski, "Macular pigment assessment using a colour monitor," Color Res. Appl. 26, S261-S263 (2001).

38. M. Greschner, J. Shlens, C. Bakolitsa, G. D. Field, J. L. Gauthier, L. H Jepson, A. Sher, A. M. Litke, and E. J. Chichilnisky, "Correlated firing among major ganglion cell types in primate retina," J. Physiol. 589, 75-86 (2011). 